\title{
Cuff-Less Blood Pressure Estimation from Electrocardiogram and Photoplethysmography Based on VGG19- LSTM Network
}

\author{
Yanan PU1 ${ }^{1}$, Xiaoxue XIE, Ling XIONG and Heng ZHANG* \\ School of Computer and Information Science, School of Software, Southwest \\ University, Chongqing, China
}

\begin{abstract}
In recent years, studies have found that the hierarchical neural network with LSTM network has higher accuracy than another feature engineering. Therefore, this paper first tries to build a multi-stage blood pressure estimation model through VGG19 and LSTM network. Based on the time node of the R wave peak in the QRS waveform in ECG, VGG19 is used to extract various higherdimensional and rich life characteristics in the PPG signal segment by heartbeat as the unit and focus on processing the dynamics of SBP and DBP Correlation, finally use the LSTM model to extract the time dependence of the vital signs. Results: Experiments show that compared with similar multi-stage models, this model has higher accuracy. The performance of this method meets the Advancement of Medical Instrumentation (AAMI) standard and reaches the A level of the British Hypertension Society (BHS) standard. The average error and standard deviation of the estimated value of SBP were $1.73504 .9606 \mathrm{mmHg}$, and the average error and standard deviation of the estimated value of DBP were $0.78392 .7700 \mathrm{mmHg}$, respectively.
\end{abstract}

Keywords. Blood pressure, photoplethysmography, multistage model, VGGNet, long short-term memory

\section{Introduction}

\subsection{Background}

Blood pressure refers to the lateral pressure acting on the wall of a blood vessel in the process of blood flow, which has significant physiological significance. According to the statistical report of the World Health Organization [1], in 2014, more than one fifth ( $24.0 \%$ for men and $20.5 \%$ for women) of adults over the age of 18 in the world were affected by hypertension. According to the updated results on The Lancet in 2017 [2], high blood pressure affects 1.13 billion people worldwide, of which more than half of adults with high blood pressure live in Asia.

\footnotetext{
* Corresponding Author, Heng ZHANG, School of Computer and Information Science, School of Software, Southwest University, Chongqing, China; Email: dahaizhangheng@swu.edu.cn.

${ }^{1}$ First Author, Yanan PU, School of Computer and Information Science, School of Software, Southwest University, Chongqing, China; Email: 312715907@qq.com.
} 
Although hypertension is preventable and controllable, most patients do not know that they are sick and fail to receive timely treatment. The control rate is meager. However, the real-time monitoring of blood pressure is necessary for the prevention, early detection, and control of hypertension. At present, the "gold standard" of blood pressure measurement is invasive, most of which are only carried out in the operating room and intensive care unit and are not suitable for daily home care. The existing continuous blood pressure measurement devices based on body surface physiological signals also need to wear sleeves. The current serial blood pressure measurement devices based on physiological signals on the body surface also need to wear cuffs and need to be calibrated at intervals. The patients must pay attention to keep still all the time, which undoubtedly makes the patients very uncomfortable [3-10]. With the development of wearable sensors, many scholars have done a lot of research on non-invasive continuous blood pressure estimation using various human life parameters in recent years. More and more studies have proved that pulse transmission time (PTT) can be used as the basis for non-invasive, cuffless, and continuous blood pressure measurement [11]. Studies have shown that PTT is inversely proportional to BP [12], as shown in the following formula (1) As shown, where 1 is the length of the arterial tube wall transmitted by the pulse wave, $\mathrm{L}=\rho / \mathrm{A}, \rho$ is the blood density, $\mathrm{A}$ is the cross-sectional area of the artery, and $\mathrm{L}$ is a constant, which represents the arterial inertia per unit length. The elasticity of the tube is flexible. The degree $\mathrm{C}$ is shown in formula (2), where $\mathrm{h}$ is the wall thickness and $\mathrm{E}$ is the elastic modulus of the tube wall, which increases with the increase of pressure P. As shown in formula (3), E0 is a constant, corresponds to the value of the modulus of elasticity under no pressure, $\alpha$ is the characteristic parameter of the arterial blood vessel, and $\mathrm{p}$ is the pressure on the arterial blood vessel.

$$
\begin{gathered}
\mathrm{PTT}=1 \sqrt{L C(P)} \\
\mathrm{C}=2 \pi r^{3} /(E * h) \\
\mathrm{E}(\mathrm{P})=E_{0}{ }^{\alpha P}
\end{gathered}
$$

\subsection{Research Status}

In 1981, Geddes et al. [13] first proposed the interdependence between BP and PPT. They obtained PTT signals from different parts of 10 anesthetized dogs and found that blood pressure was inversely proportional to PTT. After that, the researchers found the inverse relationship between PTT and blood pressure and proposed a linear model [14], which used PPT to predict blood pressure. In 2005, PON et al. [15] proposed the nonlinear relationship between PTT and blood pressure and proposed a nonlinear model based on PTT. In 2013, Liu Songsong [16] proposed to add heart rate variability as a compensation item while studying the relationship between PTT and blood pressure. This idea makes the detection result of diastolic blood pressure more accurate. So it can be estimated simply by indicating the relative timing between the proximal and distal waveforms of the arterial pulse. Therefore, PTT can realize non-invasive, automatic, and sleeveless blood pressure monitoring.

Nowadays, more and more scholars choose to use Photoplethysmography (PPG) [1 7-19], which is low-cost and straightforward, to study blood pressure. The principle is $t$ o detect changes in blood volume in living tissues by photoelectric means, and there are 
many researchers. Use PPG signals to determine PTT, and study the critical informatio $\mathrm{n}$ of PPG [20-23]. The physical basis of photoplethysmography measurement is Beer-L ambert's law, and the formula is as follows:

$$
\mathrm{A}=\log _{10}\left(\frac{I_{\text {out }}}{I_{\text {in }}}\right)=\log _{10}\left(\frac{1}{T}\right)=L \times b \times c
$$

In the formula, $\mathrm{A}$ is the absorbance, $\mathrm{I}_{\text {in }}$ represents the incident light intensity, $\mathrm{I}_{\text {out }}$ represents the output light intensity, $\mathrm{T}$ is the ratio of the incident light intensity to the output light intensity, that is, the transmittance, $\mathrm{K}$ is the absorption coefficient, $\mathrm{b}$ is the thickness of the light-absorbing medium, and $\mathrm{c}$ Is the concentration of the medium.

Although the composition of PPG has not been studied, like the heartbeats, changes in the blood volume in the blood vessels will cause corresponding changes in the absorption of light by the blood so that the PPG signal contains valuable cardiovascular system information. PTT, pulse wave velocity (PWV), and pulse arrival time (PAT) are the most commonly used features for continuous blood pressure estimation. Of course, PWV can be calculated by PTT [24]. PTT is the time delay of pressure wave propagation between two arterial parts. The pressure wave can be seen as an acute expansion of the arterial wall, which usually moves much faster than blood [25]. PTT is generally negatively correlated with BP and can be estimated simply by indicating the relative timing between the proximal and distal waveforms of the arterial pulse. In 2015, Mukkamala et al. [11] proposed two arterial wall mechanics and wave propagation models in the artery. The arterial wall model established the relationship between blood pressure and arterial elasticity. The arterial wave propagation model shows the relationship between arterial elasticity and PTT. The model concludes that PTT can effectively monitor BP when the following conditions are met:

(a) Smooth muscle contraction and viscous effects can be ignored.

(b) Aging and dis-ease will not change arterial elasticity.

(c) No wave reflection interference.

In 2015, Ding et al. [20] proposed that PTT has a strong correlation with SBP. However, there are many other factors besides PTT that are also related to BP. It is difficult to establish a model to measure blood pressure only through PTT and BP. The accuracy is not high, and the universality is also flawed.

The morphological characteristics of the PPG signal will change drastically with certain diseases or aging, which makes the morphological characteristics of the manually extracted PPG signal unreliable. Therefore, many researchers have shifted their research focus to using machine learning methods to remove relevant features of PPG signals. Tanveer et al. [21] proposed a wave-based hierarchical artificial neural network-long short-term memory (ANN-LSTM) model for BP estimation. ANN is used to extract morphological features from PPG and ECG, and then LSTM is used to interpret the temporal changes of the features. Chen et al. [26] used K-nearest-neighbor (KNN) to establish a blood pressure prediction model by extracting nine morphological features from each PPG cycle signal. Senturk et al. [27] used PPG and ECG to estimate blood pressure through a recurrent neural network (RNN), nonlinear autoregressive neural network (NARX-NN), and long short-term memory neural network (LSTM-NN). Esmaelpoor et al. [22] first extracted the morphological features of the PPG signal through CNN. They estimated SBP and DBP, respectively, and then captured the time dependence through LSTM while considering the dynamic relationship between systolic and diastolic blood pressure. Harfiya et al. [28] learned the signal conversion from PPG 
to arterial blood pressure (ABP) through a deep learning model and then extracted the values of SBP and DBP. Sun et al. [29] used CNN based on Hilbert-Huang Transform (HHT) to predict blood pressure through PPG signals.

\subsection{Contribution}

This paper proposes a new multi-stage model based on deep neural networks. Through experiments, we found that the more profound the network, the accuracy of blood pressure estimation results may not necessarily improve. The traditional VGG network cannot meet our needs. Therefore, we use the improved VGG model to train relatively more from the PPG signal. Fewer parameters extract higher latitude and more special abstract features and estimate systolic and diastolic blood pressure. It has higher accuracy than similar multi-stage models and reduces the error gap between SBP and DBP estimation based on PPG signal to a certain extent. It is feasible to use machine learning technology to determine the mapping relationship between input and output and the representation itself. And often, low-level neural network models are likely to ignore the high-dimensional features in the input data, but deep learning can express complex expressions through other simple representations, solving the core problem in representation learning, another achievement of deep learning. It is an extension of reinforcement learning and can extract high-dimensional, high-information probability from simple concepts. Therefore, deep learning is suitable for solving this type of problem [30]. In recent years, due to the increasing amount of data and model scale, the development of deep learning has been promoted, and it has also been widely used in non-computer fields such as biology, medical treatment, and finance [31-33].

\subsection{Paper Structure}

The structure of other parts of this article is as follows: Chapter 2 mainly describes our data source, preprocessing steps and model organization structure. Chapter 3 mainly introduces our experimental results. Chapter 4 mainly discusses and summarizes the full text.

\section{Materials and Method}

This chapter will introduce in detail the sources and types of data, the preprocessing process and the proposed model.

\subsection{Data Sources}

This article uses MIT's Multiparameter Intelligent Monitoring data in the intensive care II (MIMIC-II) database [34] for research. Before obtaining the data, I completed the relevant courses of the CITI project and received relevant certifications. MIMIC-II contains various parameter data of more than 15,000 ICU patients. From its sub-data set [3], we extracted 114 men, 86 women, and a total of 200 patients' complete PPG, ECG, $\mathrm{ABP}$ records. Their average age is 61.6 years, and the standard deviation of age is \pm 14.6 years. A finger pulse oximeter collects the PPG signal, and an invasive sphygmomanometer manages ABP. The sampling frequency of all signals is $125 \mathrm{~Hz}$. 


\subsection{Pretreatment Steps}

(1) The original ECG and PPG signals contain various low-frequency baseline drift and respiratory components, high-frequency power line harmonics, and EMG signal artifacts. These interferences or noises can be suppressed by filtering. In this paper, aiming at the frequency domain of noise, the frequency band of $0.48 \mathrm{~Hz}-40 \mathrm{~Hz}$ is selected for BP estimation through a band-pass filter. And add the $0 \mathrm{~Hz}-0.05 \mathrm{~Hz}$ direct current $\mathrm{DC}$ value to the PPG signal through a low-pass filter to obtain the final filtered version of the PPG signal.

(2) Since whether the QRS complex can be accurately detected directly determines the accuracy of the result, this paper adopts the method based on the extensive similarity of the integrated linear prediction residual (ILPR) in the ECG structure and the speech signal, and the QRS complex in the ECG The appearance of R waves is used as a time reference [35-37]. The advantage of this method is that it avoids the routine use of thresholds for operation. According to ECG, the PPG signal segment of each heartbeat and the corresponding SBP and DBP standard values were extracted from the PPG and ABP waveforms as the input and target values of the model, respectively. The data of 49612 heartbeats were extracted in this study. Finally, the collected PPG signal is normalized. The histogram of the extracted standard values of SBP and DBP is shown in figure 1. The average values of SBP and DBP are $121.333 \mathrm{mmHg}$ and $60.7784 \mathrm{mmHg}$, respectively, and the standard deviations are \pm 18.9004 and \pm 9.2324 , respectively. We will use these extracted data for further research.
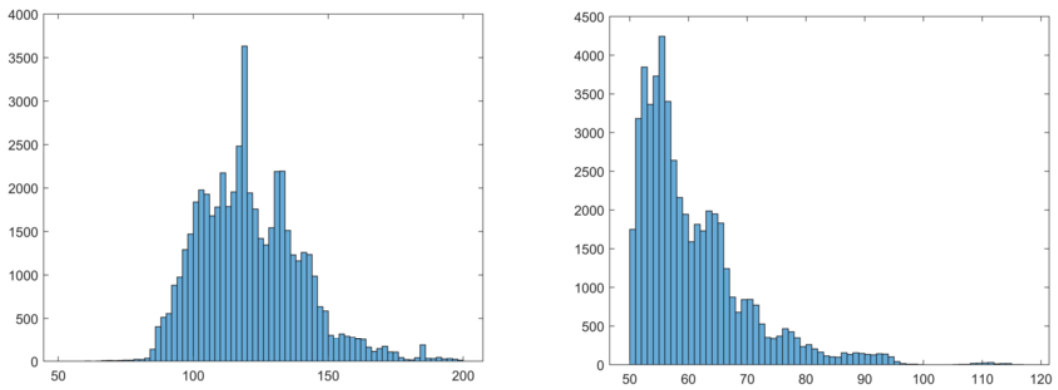

Figure 1. Histogram of SBP(left) and DBP(right) standard values.

(3) According to the above method, we divide the PPG signal into several segments, each segment represents a heartbeat sequence, and each sequence contains 250 data. When the data volume of the segmented PPG segment is less than 250 data, it will be expanded by zero padding. When the amount of data in the PPG segment is greater than 250 data, the redundant data is removed and adjusted to 250 data. The purpose of this is to be compatible with the complete PPG data containing most of the heartbeats. At this time, every The PPG signal of each heartbeat is expressed as $X_{1}, X_{2}, \ldots X_{i}$, and the standard values of SBP and DBP extracted from ABP for each heartbeat are constructed as an array $\mathrm{Y}_{1}, \mathrm{Y}_{2}, \ldots \mathrm{Y}_{\mathrm{i}}$, each $\mathrm{Y}$ element is a two-dimensional vector, $\mathrm{Ie}_{\mathrm{i}}=\left\{\mathrm{SBP}_{\mathrm{i}}, \mathrm{DBP}_{\mathrm{i}}\right\}$, where $i$ represents the number of cycles of the PPG signal, $\mathrm{SBP}_{\mathrm{i}}$ represents the target value of systolic blood pressure, and the target value of $\mathrm{DBP}_{\mathrm{i}}$ diastolic blood pressure. Finally, the array $\{Y\}$ is used as the target sequence of the BP estimation process, the entire preprocessing step as shown in figure 2. 


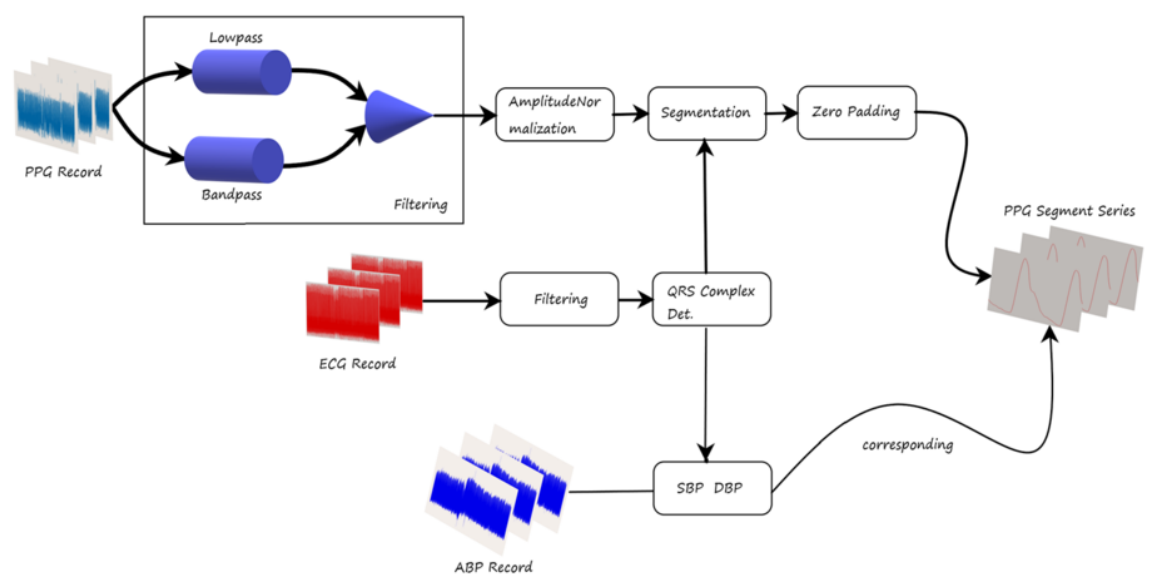

Figure 2. Preprocessing steps and segmentation extraction process.

\subsection{Model}

The overall framework of the model is shown in figure 3. The whole model is divided into two parts, and each part is composed of an adjusted VGG network model and an LSTM network model. The primary function of the upper part is the estimation of SBP, and the primary function of the lower part is the estimation of DBP.

The first neural network of each part is the adjusted VGG19 network model. The detailed information of the adjustment will be described in section 3.1. There is no essential difference between VGG19 and VGG16, but the depth is different. The network model is a variant of the convolutional neural network. It is related work on ILSVRC 2014. It mainly proves that increasing the depth of the network can affect the network to a certain extent. The final performance. Using a stacked small convolution kernel is better than using a large convolution kernel because multiple non-linear layers can increase the depth of the network to ensure that more complex patterns are learned, and the cost is relatively small (fewer parameters) [38]. Various studies have shown that the relationship between PPG signal and DBP is more prominent. In other words, it is more accurate to use a PPG signal to estimate the value of DBP [22, 27, 29]. As shown in figure 1 , the fluctuation of SBP is usually more significant than DBP fluctuates a lot, and the estimation is more complicated. Therefore, the adjusted VGG19 (VGG19 has a higher depth than VGG16) is used to extract the higher-dimensional features of the PPG signal at less cost to learn the higher-dimensional and more complex mapping relationship between the SBP and the PPG signal. In this study, the adjusted VGG19 model has 16 convolutional layers, the output of the 16th convolutional layer is used as the extracted feature vector, and the output of the entire network is used as the staged estimate. Considering the relationship between SBP and DBP [7, 39], we refer to Ref. 25 and take the phase estimation of DBP as the extended filling of the SBP feature vector. Similarly, we take the phase estimation of SBP as the ample filling of the DBP feature vector and then use it as the input vector of LSTM for the final BP estimation. 


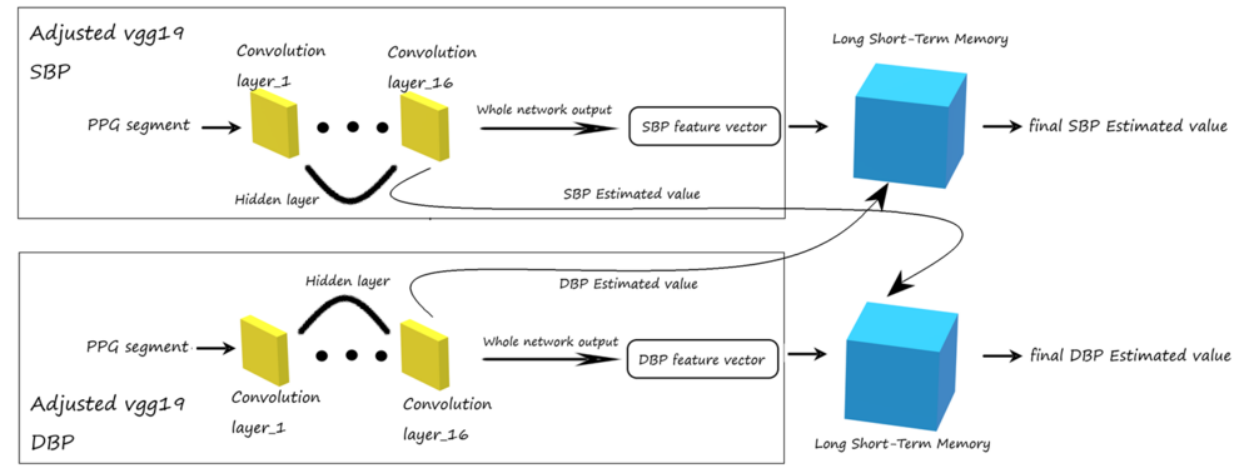

Figure 3. Overall framework of the model.

The second neural network in each part is an LSTM network stacked by two lstmLayer and two fully-connected-Layer. The LSTM network is a particular structure of the RNN network, which can learn long-term dependence information, that is, the long-term dependence relationship of the input vector [40]. So we use the LSTM network to estimate the blood pressure value based on its characteristics and the adjusted features extracted by the VGG network, so the time change of the PPG signal segment can be well learned by the LSTM network so that it can be estimated based on all previous PPG signal segments The current blood pressure value.

\section{Experimental Result}

\subsection{Model}

In the first part, two adjusted VGG19 models are used to estimate the corresponding SBP and DBP values, and the characteristic vectors of systolic and diastolic blood pressure are extracted. Through continuous testing, finally selected parameters, as shown in figure 4 , the input is a $1 \times 250 \times 1$ PPG signal segment, each VGG contains a total of 5 convolution-al layer stacks, a total of 16 convolutional layers, all convolutional layers The size of the convolution kernel is $1 \times 5$, but the number of convolution kernels in each convolution layer stack is different, which are $8,16,32,64,32$. After each convolution layer stack, a $1 \times 3$ average pooling layer with a step size of 2 . Compared with the original VGG19 net-work model, all hidden layers also contain a linear normalization layer and a ReLU layer. The last is a channel with 1 Fully connected layer because the VGG19 model includes a total of 3 fully connected layers, which causes the model to use too many parameters. Still, we found through experiments that removing the two layers of fully connected does not have too much impact on performance, so after adjustment, The VGG19 model reduces two fully connected layers than the original model. In this study, the output of the 16th convolutional layer containing 448 elements is used as the extracted feature vector, and the output of the entire network is used as a staged BP estimate to expand the feature vector. Finally, a vector containing 449 elements is used as the following step Input to the LSTM network. To compare the performance of our model and the traditional CNN model, we reproduced the experiments of other researchers, and the experimental results will be introduced in detail in section 3.3. 


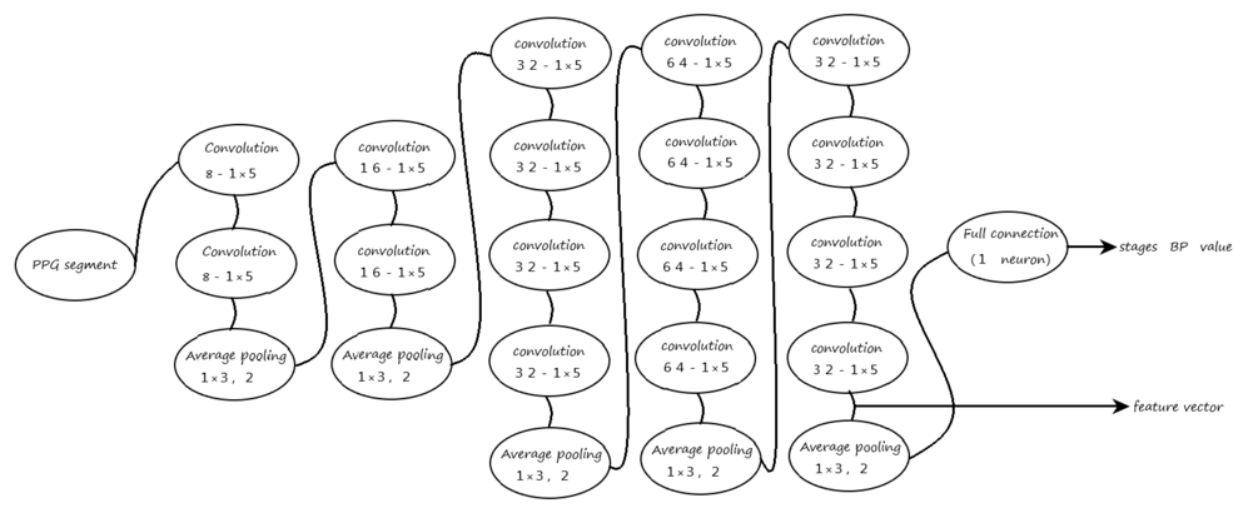

Figure 4. Proposed architecture for each VGG19 network in part one.

\section{2. $L S T M$}

In the second part, two stacked long and short-term memory networks are used to estimate the final BP value. As shown in figure 5, after many experiments, the first layer of LSTM is finally determined as 64 units, and the second layer is selected as 32 units. The input of this part is the feature vector containing 448 elements described in Section 3.1. In addition, because the relationship between PPG and BP is not unique, the blood pressure value of the same PPG shape is not necessarily the same [22, 23]. Therefore, we use the cropping technique to extend the training set [21, 22], and the experiment proves that the data set enhanced by this method can better describe the possible timedomain relationship.

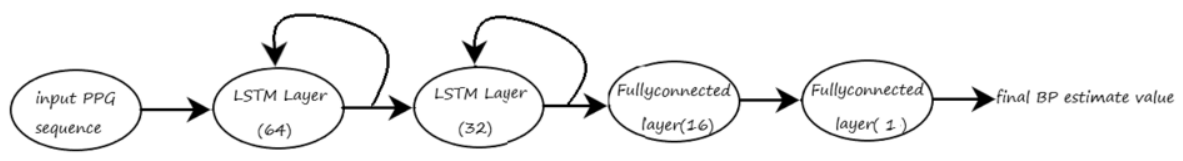

Figure 5. Proposed architecture for each LSTM network in part two.

\subsection{Model Performance}

As shown in table 1, we have compared the performance of 4-layer convolutional CNN, VGG, and our model through experiments. We can find that the version of the unadjusted VGG model is even worse than that of 4-layer convolutional CNN. By adjusting the VGG model, all aspects of its performance have been greatly improved, and the standard deviations of SBP and DBP have been down from $7.9135 \mathrm{mmHg}$ and $3.6505 \mathrm{mmHg}$ to $5.9216 \mathrm{mmHg}$ and $2.9902 \mathrm{mmHg}$, respectively.

Table 1. The performance of our model, CNN and VGG19.

\begin{tabular}{|c|c|c|c|c|c|c|c|c|}
\hline Method & $\begin{array}{l}\text { SBP } \\
\text { RMSE } \\
\end{array}$ & $\begin{array}{l}\text { SBP } \\
\text { MAE } \\
\end{array}$ & $\begin{array}{l}\text { SBP } \\
\text { ME } \\
\end{array}$ & \begin{tabular}{|l|} 
SBP \\
STD \\
\end{tabular} & $\begin{array}{l}\text { DBP } \\
\text { RMSE } \\
\end{array}$ & $\begin{array}{l}\text { DBP } \\
\text { MAE } \\
\end{array}$ & \begin{tabular}{|l} 
DPB \\
ME \\
\end{tabular} & $\begin{array}{l}\text { DBP } \\
\text { STD } \\
\end{array}$ \\
\hline $\mathrm{CNN}$ [22] & 7.8519 & 5.3562 & -0.1269 & 7.8513 & 3.3930 & 2.3544 & -0.1119 & 3.3913 \\
\hline VGG19 & 7.9618 & 5.7956 & -0.0935 & 7.9135 & 3.6728 & 2.4563 & -0.4049 & 3.6505 \\
\hline $\begin{array}{l}\text { Adjusted } \\
\text { VGG19 }\end{array}$ & 5.9237 & 3.6467 & -0.3667 & 5.9216 & 3.0105 & 2.0613 & -0.3509 & 2.9902 \\
\hline
\end{tabular}


The box-and-whisker plots of the three methods are shown in figure 6 , and the mean and shape of the error distribution of the three methods can be clearly seen. Figure 7 shows the regression curves of the three methods for SBP estimation, and figure 8 shows the regression curves of the three methods for DBP estimation. According to the Pearson's correlation coefficient $\left(\mathrm{R}=\operatorname{cov}<\mathrm{X}, \mathrm{Y}>/ \sigma_{\mathrm{X}} \cdot \sigma_{\mathrm{Y}}\right)$, it can be seen that the difference in performance results in a different $\mathrm{R}$ value. The higher the performance, the greater the $\mathrm{R}$ value. After adding the LSTM network to our model, we give the Bland-Altman diagrams of the final estimation results of SBP and DBP, as shown in figure 9.
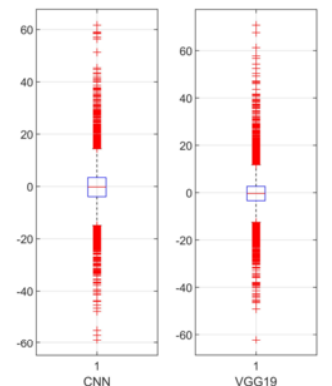

(a)

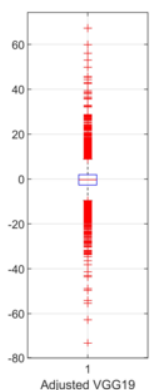

Adjusted VGG19

Figure 6. The box-and-whisker plots of estimation error for (a) SBP and (b) DBP.

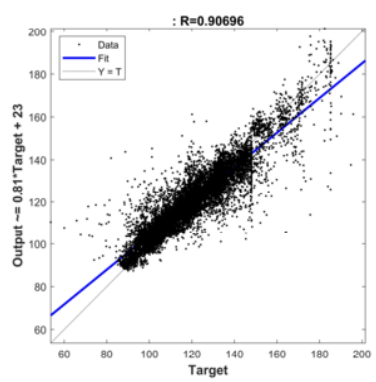

(a)

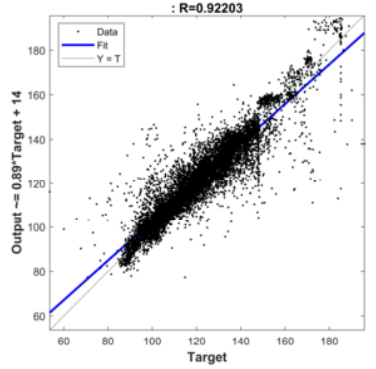

(b)

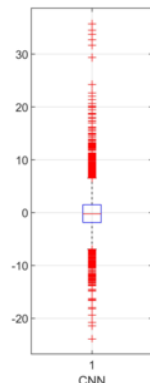

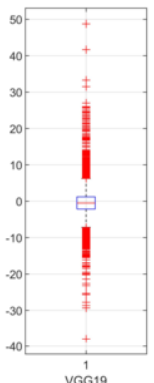

(b)

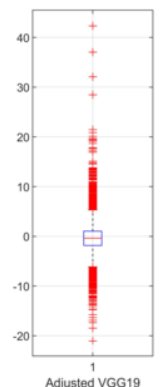

Adjusted VGG19

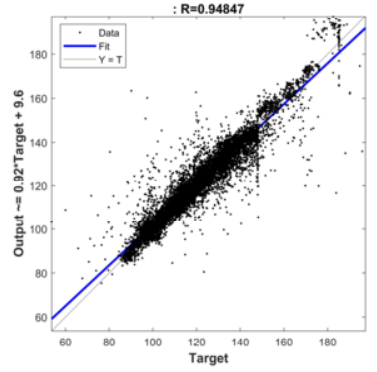

(c)

Figure 7. Comparison of SBP regression graphs of three networks (a) CNN (b) VGG19 (c) Adjusted VGG19.

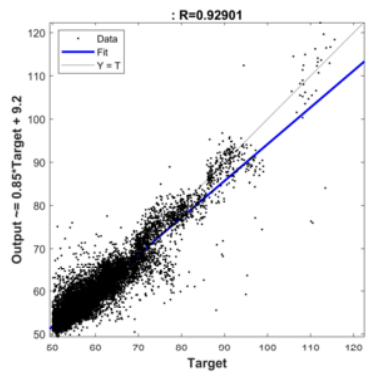

(a)

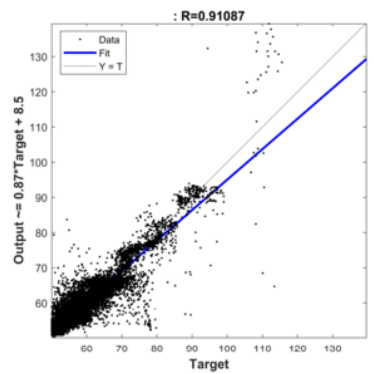

(b)

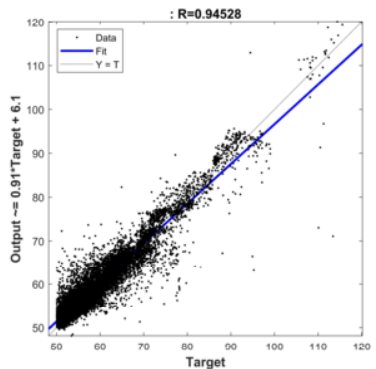

(c)

Figure 8. Comparison of DBP regression graphs of three networks (a) CNN (b) VGG19 (c) Adjusted VGG19. 


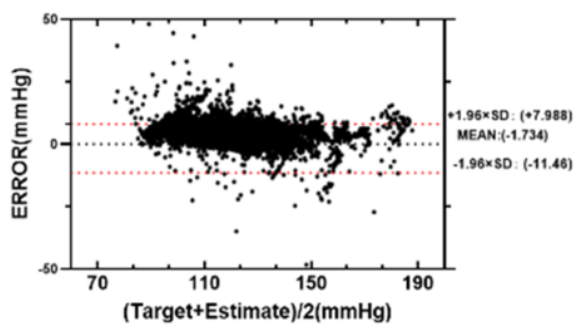

(a)

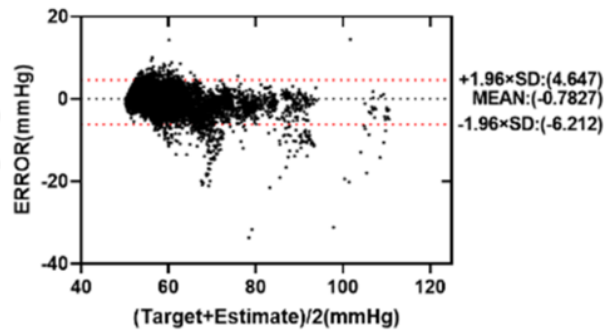

(b)

Figure 9. Bland-Altman plot of the algorithm for (a) SBP estimation and (b) DBP estimation.

\subsection{Comparison of Model Performance with British Hypertension Society (BHS) Standards}

Figure 10 shows the error distribution diagram of the final estimation result. Table 2 compares our model performance with the British Hypertension Society (BHS) standard. It can be seen that our model estimates SBP and DBP, the cumulative error frequency is less than $5 \mathrm{mmHg}$. The proportions reached $83.98 \%$ and $94.91 \%$, the ratios of less than $10 \mathrm{mmHg}$ reached $96.69 \%$ and $99.09 \%$, and the balances of less than $15 \mathrm{mmHg}$ reached $98.98 \% 99.78 \%$, all of which reached the A-level standard.

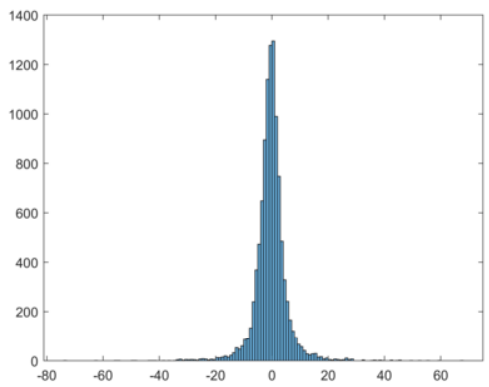

(a)

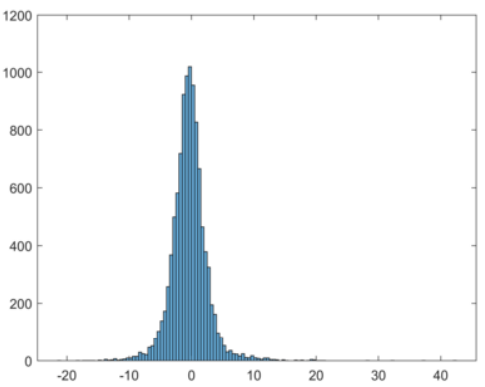

(b)

Figure 10. Error distribution of final estimation results (a) SBP estimation and (b) DBP estimation.

Table 2. Comparison of model performance with BHS standard.

\begin{tabular}{llll}
\hline Cumulative Frequency of Error & $<\mathbf{5 m m H g}$ & $<\mathbf{1 0 m m H g}$ & $<\mathbf{1 5 m m H g}$ \\
\hline Grade A & $60 \%$ & $85 \%$ & $95 \%$ \\
Grade B & $50 \%$ & $75 \%$ & $90 \%$ \\
Grade C & $40 \%$ & $65 \%$ & $85 \%$ \\
\hline Our SBP & $83.98 \%$ & $96.69 \%$ & $98.98 \%$ \\
Our DBP & $94.91 \%$ & $99.09 \%$ & $99.78 \%$ \\
\hline
\end{tabular}

\subsection{Comparison of Model Performance with Advancement of Medical Instrumentation (AAMI) Standard}

As shown in table 3, by comparing our model with the Advancement of Medical Instrumentation (AAMI) standard, the mean error (ME) and standard deviation (STD) of our model for SBP estimation are $1.7342 \mathrm{mmHg} 4.9606 \mathrm{mmHg}$, respectively, the average 
of the DBP estimation The error (ME) and standard deviation (STD) are $0.7827 \mathrm{mmHg}$ and $2.77 \mathrm{mmHg}$, respectively, which all meet the AAMI standard.

Table 3. Comparison of model performance with AAMI standard.

\begin{tabular}{llll}
\hline & ME & STD & Subject \\
\hline Estimation Error & $<5 \mathrm{mmHg}$ & $<8 \mathrm{mmHg}$ & $>85$ \\
\hline Our SBP & $1.7342 \mathrm{mmHg}$ & $4.9606 \mathrm{mmHg}$ & 200 \\
\hline Our DBP & $0.7827 \mathrm{mmHg}$ & $2.77 \mathrm{mmHg}$ & 200 \\
\hline
\end{tabular}

\subsection{Comparison with Other Models}

In order to verify the performance of our model, we reproduced the non-invasive continuous blood pressure model proposed by some researchers, as shown in table 4. All models use the same data set and standard. By comparing the amount of sample data and the root mean square error, average error, and standard deviation of the estimated BP value, the model we proposed is more universal and has better performance.

Table 4. Comparison with other model.

\begin{tabular}{llllllll}
\hline Method & \multirow{2}{*}{ Subject } & $\begin{array}{l}\text { SBP } \\
\text { RMSE }\end{array}$ & $\begin{array}{l}\text { SBP } \\
\text { ME }\end{array}$ & $\begin{array}{l}\text { SBP } \\
\text { STD }\end{array}$ & $\begin{array}{l}\text { DBP } \\
\text { RMSE }\end{array}$ & $\begin{array}{l}\text { DBP } \\
\text { ME }\end{array}$ & $\begin{array}{l}\text { DBP } \\
\text { STD }\end{array}$ \\
\hline Regression [20] & 27 & - & -0.39 & 5.04 & - & -0.03 & 4.26 \\
\hline Linear Regression [23] & 22 & - & 1.32 & 1.03 & - & 0.93 & 0.84 \\
\hline Machine Learning [41] & 51 & 11.23 & - & - & 18.74 & - & - \\
\hline CNN-LSTM [22] & 200 & 6.1 & 0.84 & 6.04 & 3.12 & 0.97 & 2.84 \\
\hline Our result & 200 & 5.25 & 1.73 & 4.96 & 2.85 & 0.78 & 2.77 \\
\hline
\end{tabular}

\section{Discussion and Conclusion}

Our research estimates SBP and DBP by replacing CNN, RNN, or other low-depth networks with adjusted VGG networks and uses deeper neural networks to extract higher-latitude features in PPG signals. The model refers to the two independent paths and multi-stage models proposed by other researchers so that each direction in the model architecture can be independently extracted and estimated. This method improves the sensitivity of the model while taking into account the correlation between SBP and DBP. The estimated value of the first-stage model is used as the extracted feature. Then it is extended to the feature vector of another path and used as the input of the second-stage LSTM network model. The first stage of the model emphasizes local high-dimensional features extracted through multi-layer convolution. The second stage emphasizes timedomain correlation, which strengthens this connection through planting technology.

Traditional hand co-extraction features will be affected by age changes or diseases. The drastic changes in the PPG signal will cause the hand co-extraction features to be less credible, so we use machine learning to extract high-dimensional features, which is more robust.

$\mathrm{CNN}$ can extract features at different scales and share weights. Compared with a fully connected network, it reduces memory usage, but VGG can learn characteristics of higher dimensions at a more negligible cost. Under the premise of the same convolutional field of view, there are fewer parameters to learn. 
LSTM can determine the time-domain changes of the PPG signal, thereby improving the accuracy of the model. Because LSTM uses feedback connections to memorize the representation of the current event, this solves the shortcoming that manual features cannot extract long-term dependencies.

Through the combination of the two network models, our model accuracy has been further improved. Both BHS and AAMI standards have proven the accuracy of our model.

In addition, although we have achieved the extraction of higher-dimensional features at a more negligible cost by increasing the number of network layers and reducing the size of the convolution kernel, it still reduces the operating efficiency of the model. You can reduce the number of network layers to ensure a sure accuracy. In the case of the operating efficiency of the model is further improved, and at the same time, some reasonable manual features can also be added to expand the feature vector extracted by machine learning so as to select a better feature set.

\section{Acknowledgment}

This work was supported by the Fundamental Research Funds for the Central Universities, China, under grant XDJK2020B029. And this work was supported by the Major Transverse Project, China, under Grant SWU41015718 and Grant SWU20710953.

\section{References}

[1] World Health Organization. World health statistics 2015. World Health Organization, 2015.

[2] Bin Zhou, James Bentham, Mariachiara Di Cesare, Honor Bixby, Goodarz Danaei,Melanie J Cowan, Christopher J Paciorek, Gitanjali Singh, Kaveh Hajifathalian, James E Bennett, et al. Worldwide trends in blood pressure from 1975 to 2015: a pooled analysis of 1479 population-based measurement studies with 19. 1 million participants. The Lancet, 389(10064):37-55, 2017.

[3] Mohamad Kachuee, Mohammad Mahdi Kiani, Hoda Mohammadzade, and Mahdi Shabany.Cuff-less high-accuracy calibration-free blood pressure estimation using pulse transit time. In 2015 IEEE international symposium on circuits and systems (ISCAS), pages 1006-1009. IEEE, 2015.

[4] Beate H McGhee and Elizabeth J Bridges. Monitoring arterial blood pressure: what you may not know. Critical care nurse, 22(2):60-79, 2002.

[5] Gregory Nuttall, Jennifer Burckhardt, Anita Hadley, Sarah Kane, Daryl Kor, Mary Shirk Marienau, Darrell R Schroeder, Kathryn Handlogten, Gregory Wilson, and William C Oliver. Surgical and patient risk factors for severe arterial line complications in adults. Anesthesiology, 124(3):590-597, 2016.

[6] Dorothee Perloff, Carlene Grim, John Flack, Edward D Frohlich, Martha Hill, Mary McDonald, and Bruce Z Morgenstern. Human blood pressure determination by sphygmomanometry. Circulation, 88(5):2460-2470, 1993.

[7] Gary Drzewiecki, Rebecca Hood, and H Apple. Theory of the oscillometric maximum and the systolic and diastolic detection ratios. Annals of biomedical engineering, 22(1):88-96, 1994.

[8] KH Wesseling. Physiocal, calibrating finger vascular physiology for finapres. Homeostasis, 36:67-82, 1995.

[9] Gary M Drzewiecki, Julius Melbin, and Abraham Noordergraaf. Arterial tonometry: review and analysis. Journal of biomechanics, 16(2):141-152, 1983.

[10] Elliott S Greene and John I Gerson. Arterial pulse wave velocity: a limited index of systemic vascular resistance during normotensive anesthesia in dogs. Journal of clinical monitoring, 1(4):219-226, 1985.

[11] Ramakrishna Mukkamala, Jin-Oh Hahn, Omer T Inan, Lalit K Mestha, Chang-Sei Kim, Hakan T"'oreyin, and Survi Kyal. Toward ubiquitous blood pressure monitoring via pulse transit time: theory and practice. IEEE Transactions on Biomedical Engineering, 62(8): 1879-1901, 2015.

[12] Ramakrishna Mukkamala and Jin-Oh Hahn. Toward ubiquitous blood pressure monitoring via pulse transit time: Predictions on maximum calibration period and acceptable error limits. IEEE Transactions on Biomedical Engineering, 65(6):1410-1420, 2017. 
[13] LA Geddes, MH Voelz, CF Babbs, JD Bourland, and WA Tacker. Pulse transit time as an indicator of arterial blood pressure. psychophysiology, 18(1):71-74, 1981.

[14] KW Chan, K Hung, and YT Zhang. Noninvasive and cuffless measurements of blood pressure for telemedicine. In 2001 Conference Proceedings of the 23rd Annual International Conference of the IEEE Engineering in Medicine and Biology Society, volume 4, pages 3592-3593. IEEE, 2001.

[15] CCY Poon and YT Zhang. Cuff-less and noninvasive measurements of arterial blood pressure by pulse transit time. In 2005 IEEE engineering in medicine and biology 27 th annual conference, pages $5877-$ 5880. IEEE, 2006.

[16] Liu Songsong. Research and implementation of wearable continuous blood pressure monitoring system. $\mathrm{PhD}$ thesis, Harbin Institute of Technology, 2013.

[17] Mark Wong Kei Fong, EYK Ng, Kenneth Er Zi Jian, and Tan Jen Hong. Svr ensemblebased continuous blood pressure prediction using multi-channel photoplethysmogram. Computers in biology and medicine, 113:103392, 2019.

[18] Christina Orphanidou. Derivation of respiration rate from ambulatory ecg and ppg using ensemble empirical mode decomposition: Comparison and fusion. Computers in biology and medicine, 81:45-54, 2017.

[19] Wan-Hua Lin, Hui Wang, Oluwarotimi Williams Samuel, Gengxing Liu, Zhen Huang, and Guanglin Li. New photoplethysmogram indicators for improving cuffless and continuous blood pressure estimation accuracy. Physiological measurement, 39(2):025005, 2018.

[20] Xiao-Rong Ding, Yuan-Ting Zhang, Jing Liu, Wen-Xuan Dai, and Hon Ki Tsang. Continuous cuffless blood pressure estimation using pulse transit time and photoplethysmogram intensity ratio. IEEE Transactions on Biomedical Engineering, 63(5):964-972, 2015.

[21] Md Sayed Tanveer and Md Kamrul Hasan. Cuffless blood pressure estimation from electrocardiogram and photoplethysmogram using waveform based ann-lstm network. Biomedical Signal Processing and Control, 51:382-392, 2019.

[22] Jamal Esmaelpoor, Mohammad Hassan Moradi, and Abdolrahim Kadkhodamohammadi. A multistage deep neural network model for blood pressure estimation using photoplethysmogram signals. Computers in Biology and Medicine, 120:103719, 2020.

[23] Mohammad Kachuee, Mohammad Mahdi Kiani, Hoda Mohammadzade, and Mahdi Shabany. Cuffless blood pressure estimation algorithms for continuous health-care monitoring. IEEE Transactions on Biomedical Engineering, 64(4):859-869, 2016.

[24] Dilpreet Buxi, Jean-Michel Redout'e, and Mehmet Rasit Yuce. A survey on signals and systems in ambulatory blood pressure monitoring using pulse transit time. Physiological measurement, 36(3):R1, 2015.

[25] Charalambos Vlachopoulos, Michael O'Rourke, and Wilmer W Nichols. McDonald's blood flow in arteries: theoretical, experimental and clinical principles. CRC press, 2011.

[26] Chen Yi, Cheng Jian, and Ji Wenqiang. Continuous blood pressure measurement based on photoplethysmography. In 2019 14th IEEE International Conference on Electronic Measurement \& Instruments (ICEMI), pages 1656-1663. IEEE, 2019.

[27] Umit Senturk, Kemal Polat, and Ibrahim Yucedag. A non-invasive continuous cuffless blood pressure estimation using dynamic recurrent neural networks. Applied Acoustics, 170:107534, 2020.

[28] Latifa Nabila Harfiya, Ching-Chun Chang, and Yung-Hui Li. Continuous blood pressure estimation using exclusively photopletysmography by lstm-based signal-to-signal translation. Sensors, 21(9):2952, 2021.

[29] Xiaoxiao Sun, Liang Zhou, Shendong Chang, and Zhaohui Liu. Using cnn and hht to predict blood pressure level based on photoplethysmography and its derivatives. Biosensors, 11 (4):120, 2021.

[30] Yann LeCun, Yoshua Bengio, and Geoffrey Hinton. Deep learning. nature, 521(7553): 436-444, 2015.

[31] Peter Stenvinkel, Colin J Meyer, Geoffrey A Block, Glenn M Chertow, and Paul G Shiels. Understanding the role of the cytoprotective transcription factor nuclear factor erythroid 2-related factor 2-lessons from evolution, the animal kingdom and rare progeroid syndromes. Nephrology Dialysis Transplantation, 35(12):2036-2045, 2020.

[32] Alfredo Vellido. The importance of interpretability and visualization in machine learning for applications in medicine and health care. Neural computing and applications, pages 1-15, 2019.

[33] Huiming Zhu, Chao Deng, Shengjie Yue, and Yingchun Deng. Optimal reinsurance and investment problem for an insurer with counterparty risk. Insurance: Mathematics and Economics, 61:242-254, 2015.

[34] Mohammed Saeed, Mauricio Villarroel, Andrew T Reisner, Gari Clifford, Li-Wei Lehman, George Moody, Thomas Heldt, Tin H Kyaw, Benjamin Moody, and Roger G Mark. Multiparameter intelligent monitoring in intensive care ii (mimic-ii): a public-access intensive care unit database. Critical care medicine, 39(5):952, 2011.

[35] Mintu P Turakhia, Manisha Desai, Haley Hedlin, Amol Rajmane, Nisha Talati, Todd Ferris, Sumbul Desai, Divya Nag, Mithun Patel, Peter Kowey, et al. Rationale and design of a large-scale, app-based 
study to identify cardiac arrhythmias using a smartwatch: The apple heart study. American heart journal, 207:66-75, 2019.

[36] John Allen. Photoplethysmography and its application in clinical physiological measurement. Physiological measurement, 28(3):R1, 2007.

[37] AG Ramakrishnan, AP Prathosh, and TV Ananthapadmanabha. Threshold-independent qrs detection using the dynamic plosion index. IEEE Signal Processing Letters, 21(5): 554-558, 2014.

[38] Karen Simonyan and Andrew Zisserman. Very deep convolutional networks for large-scale image recognition. arXiv preprint arXiv:1409.1556, 2014.

[39] Giuseppe Schillaci and Giacomo Pucci. The dynamic relationship between systolic and diastolic blood pressure: yet another marker of vascular aging? Hypertension research, 33(7):659-661, 2010.

[40] Sepp Hochreiter and J"urgen Schmidhuber. Long short-term memory. Neural computation, 9(8):17351780, 1997.

[41] Monika Simjanoska, Martin Gjoreski, Matja z Gams, and Ana Madevska Bogdanova. Noninvasive blood pressure estimation from ecg using machine learning techniques. Sensors, 18(4):1160, 2018. 